\title{
Fractal Characteristics of Mechanical Interface Contact Parameters
}

\author{
Yuqi Gong ${ }^{1}$, Jingfang Shen ${ }^{1, a}$, Wenwei Liu² and Ling Chen ${ }^{1}$ \\ 1 College of Science, Huazhong Agricultural University, Wuhan, Hubei, 430070, China \\ ${ }^{2}$ China Electronic Product Reliability and Environmental Testing Research Institute, 510610 Guangzhou, China
}

\begin{abstract}
The contact performance of various mechanical parts has important influence on the static, dynamic and motion response of mechanical equipment. The characterization of rough surface topography is the basis for the study of friction, wear and contact deformation between mechanical bonding surfaces. By analyzing the various models of the characteristic parameters of mechanical interface, we provide a systematic research idea for the model of mechanical joint surface in the future. For further details on rough surface profile, the G-W model is put forward. Then the M-B model perfects its lack of scale independence. The relationship between actual contact area and load is derived from the M-B model based on fractal geometry theory. These formulas are used to study the characteristic parameters of mechanical interface, and the fractal model of contact damping and loss factor is established. Since fractal parameters are not limited by sampling length and resolution of measuring instrument, the new models are more reasonable than before. However, just as the M-B model needs to be improved, the model based on this need to be further studied, and the application of the model also needs to be explored more.
\end{abstract}

\section{Introduction}

The manufacture of mechanical equipment has vital influence on the economic development and comprehensive strength of the country. Therefore, the research on the design of mechanical products has important theoretical significance and application value. The rough surface morphology plays an irreplaceable role on the contact characteristics of mechanical interface. The mechanical interface performance affects the static, dynamic and motion response of mechanical equipment. Taking the basic industry as an example, half of the stiffness of the machine tool is the stiffness of mechanical interface, and the damping of the whole machine is mostly from the combination surface damping. Consequently, it is a key issue to accurate the model of mechanical interface. According to the contact characteristic parameters of mechanical interface, lots of fractal models are presented. To clarify and understand the interconnection of these models, we give a general description of the introduction, content and development of the contact stiffness and contact damping fractal model of mechanical binding surface. Furthermore, this paper can provide a systematic research idea for the model of mechanical joint surface in the future.

\section{Model of Rough Surface Contact}

\subsection{Introduction of Surface Contact Fractal Model}

It is crucial to study the friction, wear and contact deformation of mechanical interface. To further study this topic, the researchers propose the traditional contact models. The G-W model is a statistical model proposed by Greenwood and Williamson in 1966 [1]. In this model, the height distribution of rough surface topography is the random variable. Obeying Gaussian distribution, this model follows the law of classical friction. The standard deviation of height distribution and the mean curvature radius of asperities are first used to characterize the surface morphology. This model is widely accepted and used in research. However, these studies are based on traditional quantitative statistical descriptions of surface topography. The traditional measurement results will be affected by the resolution of the measuring instrument. In other words, the description of the G-W model does not have the scale independence, and it cannot reflect the whole information of the rough surface.

\subsection{Fractal Characteristics}

In 1967, Mandelbrot proposes a mathematic mehod to measure the length of the coastline [2]. As the observation scale shrinks, the area of the coastline will repeatedly appear on the peninsula and harbor. Mandelbrot also finds a clear correlation between measurements and results [3]. Later, Richardson gives the

\footnotetext{
a Corresponding author Jingfang Shen: shenjingfang16@126.com
} 
relationship between measurement scale $\varepsilon$ and measurement results $L(\varepsilon)$

$$
L(\varepsilon)=F \varepsilon^{1-D}
$$

Where $F$ and $D$ are constants, and $D$ is called the fractal dimension, which has scale independence and can reflect the roughness information on all scales [4]. For the statistical self-similar fractal curve $D$ can be calculated by equation (1). We enlarge the rough surface topography to obtain the details. The surface profile structure is similar under different multiple. The rough surface morphology is two-dimensional fractal, and its fractal dimension is called $D_{s}$. The contour curve of surface topography is one-dimensional fractal, and its fractal dimension is called $D$. Their relation is

$$
D=D_{s}-1
$$

According to the research, the contour curves of mechanical surface morphology are presented with statistical self-affinity characteristics [5]. This feature is no longer satisfied equation (1), so we need to research new methods for calculating fractal dimension of surface profile.

\subsection{Calculation Method of Fractal Dimension}

The calculation principle of fractal dimension is to measure the similarity by measuring parameters. Therefore, different measurement parameters are selected and the methods of measuring fractal dimension of surface are different. The commonly used methods include box counting, power spectral density, structural function, root mean square, etc.

\subsubsection{Box Counting Method}

The theory of box counting is the most simple and easy to understand, but the calculation error is relatively large and is not suitable for the calculation of self-affinity surface profile [6]. Cover surface profile curve with box whose side length is equal to 1 , and then divided it into $2^{n}$ small box. Count the number $M(n)$ of small boxes that intersect surface profile. The fractal dimension $D$ is related to $M(n)$ as follows

$$
D=\lim _{n \rightarrow \infty} \frac{\ln M(n)}{n \ln 2}
$$

\subsubsection{Power Spectral Density Method}

The W-M function satisfies the mathematical properties of fractal, so $z(x)$ is used by Majumdar and Tien in 1989 to describe rough surface [7]. $z(x)$ is given by

$$
\mathrm{z}(x)=G^{D-1} \sum_{n=n_{1}}^{\infty} \frac{\cos \pi \gamma^{n} x}{\gamma^{(2-D) n}} ; 1<D<2, \gamma>1
$$

Where $D$ describes the similarity of functions on different scales. $G$ describes the magnitude of the amplitude. $V$ describes the spectrum. $V$ is usually equal to 1.5. Therefore, $D$ is one of the parameters that decide $z(x)$. So the power spectrum density method and the structure function method for solving $D$ are derived.

Auto correlation function and power spectral density describe the statistical characteristics of stochastic process from two aspects: time domain and frequency domain [8,9]. By Wiener-Khinchin theorem, auto correlation function and power spectral density function is a pair of Fourier transform. So the power spectral density of stationary random process is given by

$$
\begin{aligned}
& R(\tau)=\int_{-\infty}^{\infty} S(f) e^{j 2 \pi f \tau} d f \\
& S(f)=\int_{-\infty}^{\infty} R(\tau) e^{-j 2 \pi f \tau} d \tau
\end{aligned}
$$

On the rough surface of isotropic, its two-dimensional auto correlation function $R(\tau)$ is given by

$$
R(\tau)=\lim _{L \rightarrow \infty} \frac{1}{L} \int_{0}^{L} z(x) z(x+\tau) d x
$$

The discrete power spectral density can be obtained by taking the Fourier transform of the auto correlation function $R(\tau)$, and the approximate continuous power spectral density has shown to be

$$
S(\omega) \propto \omega^{-(5-2 D)}
$$

The slope of the fitting $\operatorname{line} \lg S(\omega)-\lg \omega$ is $\alpha$, then $D=(5+\alpha) / 2$. There are many ways to estimate the power spectrum density. In addition to the auto correlation method used here, there are also classical estimation methods such as periodic graph method, Bartlett method, and Welch method. The periodic graph method is to consider the observation data of the random sequence as a finite energy sequence, directly calculating its discrete Fourier transform and then taking the square of its amplitude and dividing the total number of observed data. This estimation method is more convenient than the calculation of auto correlation method, also known as direct method. The Bartlett method and Welch method are the improvement of the auto correlation method and the periodic graph method, and the result is more accurate. Welch method made two modifications to the Bartlett method. First, Welch selects the appropriate window function to make the spectrum estimation non-negative; secondly, there is overlap between the segments, so that the variance decreases. The power spectral density method is suitable for self-affinity fractal curve [10].

\subsubsection{Structural Function Method}

The calculation principle of structural function method is similar to power spectrum density method. It was found that the structure function of a rough surface is given as

$$
S(\tau)=c \tau^{4-2 D}
$$

The slope of the fitting $\operatorname{line} \lg S-\lg \tau$ is $\alpha$, then $D=2-\alpha / 2$. The empirical research shows that the proposed power spectrum density curve and the 
structure function curve have good linear characteristics in the high frequency band, and the linear characteristics of the low frequency band are poor. These two methods are widely used in the study of mechanical rough surface. However, compared with the structure function method, the precision of fractal dimension of engineering surface is lower.

\subsubsection{Root Mean Square Method}

GE Shirong first proposes the root mean square method in 1997 [11]. The root mean square method is to calculate its covariance $\sigma(\tau)$ in different time domain $\tau_{i}$ and return the slop $\alpha$ of the $\operatorname{line} \lg \sigma(\tau)-\lg \tau$, then $D=2-\alpha$. In 2004, ZHU Hua and GE Shirong compares the fractal representation of structural function and root mean square. It is pointed out that the calculation accuracy of these two methods is related to the fractal dimension of the contour theory, while the fractal dimension is smaller and the root mean square method is better. When fractal dimension is large, the structure function method is better. Because of the explicit physical meaning of the root mean square method, they found that for the rough surface of machining, the root mean square method is more adaptive than the structure function method, and the linear interval is wider and the characterization effect is better $[12,13]$.

\subsection{Development of Surface Contact Fractal Model}

The parameters of traditional contact model do not have scale independence, so another classical contact model is produced. The M-B model is first proposed by Majumdar and Bhushan in 1991 based on fractal theory. Like the G$\mathrm{W}$ model, the contact between rough surfaces can be reduced to the contact between rough surfaces and smooth surfaces to study the distribution of contact points. In 1982, when he studies the landform, Mandelbrot found that the island is larger than a specific area $a$ and followed that

$$
\begin{gathered}
N(A>a) \sim a^{-B}, B=D / 2 \\
N(A \geq a)=a_{L}{ }^{B} a^{-B} \\
n(a) d a=-d N=B a_{L}{ }^{B} a^{-(B+1)} d a
\end{gathered}
$$

The area of all islands $A_{l}$ is given as

$$
A_{l}=\int_{0}^{a_{L}} n(a) a d a=\frac{B}{1-B} a_{L}=\frac{D}{2-D} a_{L}
$$

Majumdar finds that the moderately enlarged mechanical surface is similar to that of an island in the ocean. Therefore, the above relationship is also applicable to the study of mechanical rough surface. So the relations are

$$
n(a)=\frac{D}{2} \cdot \frac{a^{\frac{D}{2}}}{a^{\frac{D}{2}+1}}
$$

$$
A_{r}=\int_{0}^{a_{l}} n(a) a d a=\frac{D}{2-D} a_{l}
$$

The contact area of the asperity is plastic deformation when $a<a_{c}$. The contact area of the asperity is elastic deformation when $a>a_{c}$. The critical contact area $a_{c}$ is given as

$$
a_{c}=\frac{G^{2}}{(k h / 2)^{\frac{2}{D-1}}}
$$

According to the M-B model, when the load increases, the point where the plastic deformation occurs is connected to form the elastic point. The material is deformed under the action of external forces. When the external force is removed, the deformation of the material can disappear and the original shape can be completely restored. A physical phenomenon in which the object cannot be restored after the external force is removed or disappeared is a plastic deformation. This model shows that the relation between the load $P$ and the actual contact area $A_{r}$ is $P \sim A_{r}^{(3-D) / 2}$. Where $D$ is the fractal dimension of surface profile. Experimental results also confirm its correctness.

\section{Fractal Model of Contact Stiffness of Joint Surfaces}

Majumdar found that the surface profile of the mechanical surface morphology showed statistical selfaffinity characteristics, and proposed the M-B model based on the fractal theory. The model makes up the defects that the G-W model is lack of the scale independence. In 2000, Xueliang Zhang first proposes the model of contact stiffness based on fractal theory. The dynamic model method of fixed combination surface is proposed by him. He considers the normal and tangential loads, the elastic modulus, the shear modulus, Poisson ratio and density of the materials.He simplifies the model of mechanical joint surfaces and solved the normal contact stiffness $k_{n}$ of a single asperity. It is given as

$$
k_{n}=2 E r=2 E \sqrt{\frac{a}{\pi}}
$$

The contact stiffness of all asperities on the surface is integrated. According to the equations of the contact point size distribution function in M-B model, the normal contact stiffness of the mechanical interface can be written as

$$
K_{n}=\int_{a_{c}}^{a_{l}} k_{n} n(a) d a
$$

The normal contact stiffness of a single asperity is in equation (18). $n(a)$ is in equation(14) . the critical contact area of equation (16), we can obtain the nondimensional normal contact stiffness 


$$
K_{n}^{*}=\frac{2}{\bar{\pi}} g_{1}(D) A_{r}^{* \frac{D}{2}}\left[\left(\frac{2-D}{D}\right)^{\frac{1-D}{2}} \cdot A_{r}^{* \frac{1-D}{2}}-a_{c}^{* \frac{1-D}{2}}\right]
$$

In 2002, Xueliang Zhang proposes a model of tangential stiffness of mechanical joint surface based on fractal theory [14]. The tangential contact stiffness $k_{t}$ of a single asperity is given by

$$
k_{t}=\frac{8 \bar{G} \sqrt{a}}{(2-v) \sqrt{\pi}}\left(1-\frac{1}{\mu} \frac{T}{P}\right)^{1 / 3}
$$

$k_{t}$ is exist when $\frac{T}{P}<\mu$. There is a relative slide when $\frac{T}{P} \geq \mu$. The contact stiffness of all asperities is integrated. According to the calculation formula of the contact point size distribution function in M-B model, the normal contact stiffness $K_{t}$ of the mechanical joint surfaces can be given as

$$
K_{t}=\int_{a_{c}}^{a_{l}} k_{t} n(a) d a
$$

The results of digital simulation show that the contact stiffness is positively correlated with the dimensionless method. The contact stiffness is positively correlated with the fractal dimension. The contact stiffness is negatively correlated with the dimensionless fractal roughness parameter. The combined surface dimensionless tangential stiffness is positively correlated with the dimensionless method. The contact stiffness is positively correlated with the fractal dimension. The contact stiffness is negatively correlated with the dimensionless fractal roughness parameter. The contact stiffness is negatively correlated with the tangent load. This is consistent with the empirical results.

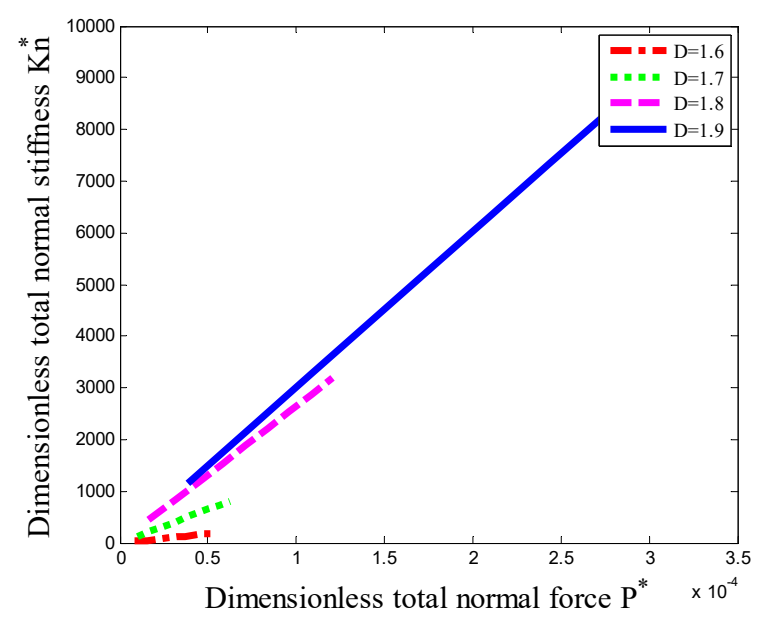

Figure 1. The influence of fractal dimension on the nondimensional normal contact stiffness

$$
\text { ( } G=10^{-9}, k=1, h=0.01 \text { ) }
$$

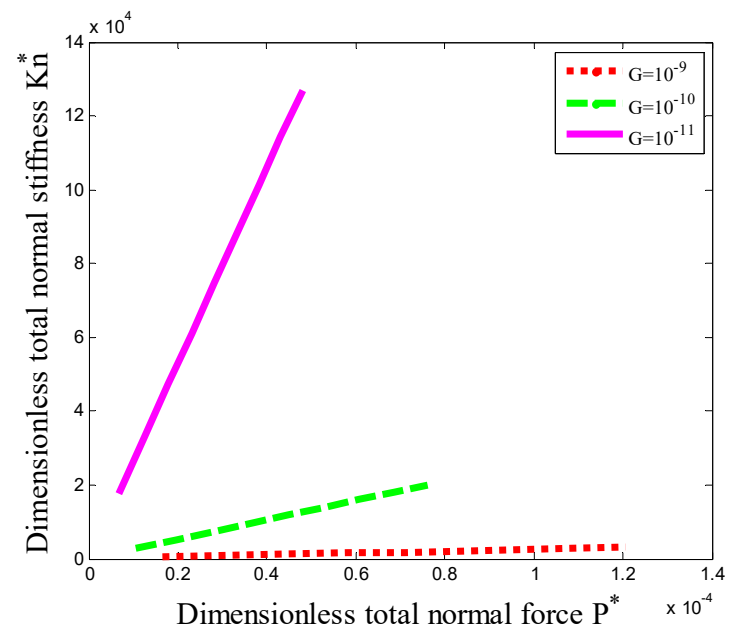

Figure 2. The influence of roughness on the non-dimensional normal contact stiffness

$$
(D=1.6, k=1, h=0.01)
$$

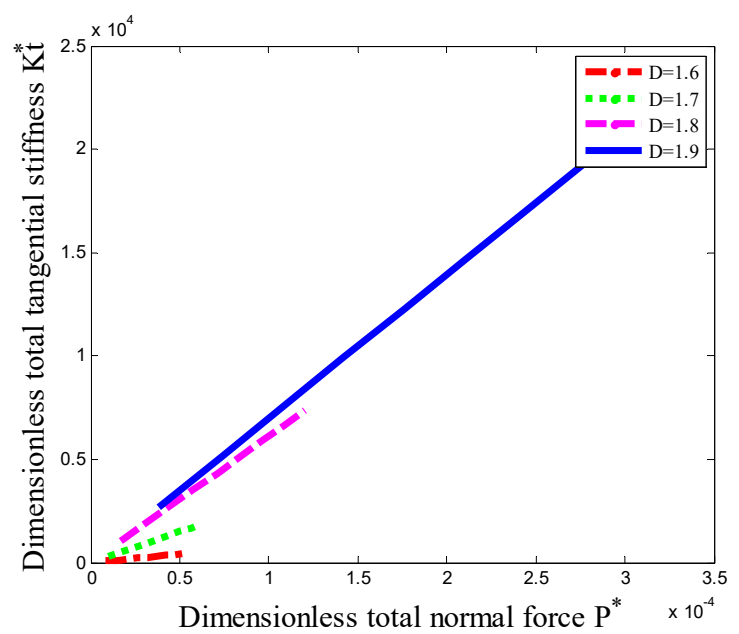

Figure 3. The influence of fractal dimension on the nondimensional tangential contact stiffness

$$
\left(G=10^{-9}, k=1, h=0.01, v=0.3, \mu=0.2\right. \text { ) }
$$

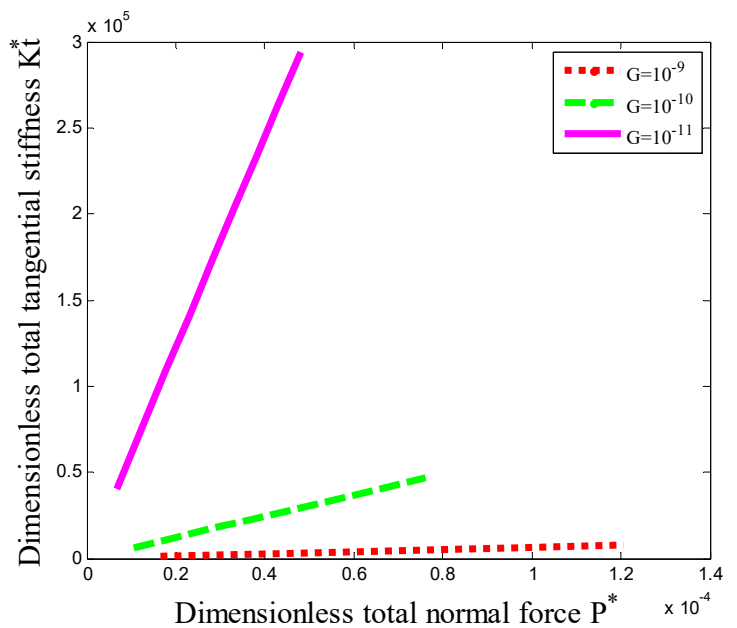

Figure 4. The influence of roughness on the non-dimensional tangential contact stiffness

$(D=1.8, k=1, h=0.01, v=0.3, \mu=0.2)$ 


\section{Conclusions}

The M-B model based on fractal theory is more advanced than the statistical G-W model. It characterizes the rough surface by fractal parameters $D$ and $G$ containing all information of surface roughness. The results of the study are independent of the resolution of the instrument. The researchers have applied the $\mathrm{M}-\mathrm{B}$ model to contact stiffness, contact damping and loss factor model, and improve it continuously. However, present models require in-depth research, and the application of the models also need to be explored:

(1) The establishment of the model only takes into consideration the various homosexual surfaces and does not involve the contact of anisotropic surfaces.

(2) The contact characteristic parameters of the combination surface include many aspects. This paper only discusses the stiffness, damping and other aspects, which is worthy of further study.

(3) When establishing a model, only one fractal parameter is considered. In addition to fractal dimension, several fractal parameters can be considered.

(4) The fractal model of mechanical interface contact characteristic parameters is based on fractal theory, assuming that the rough peak and the number of contact points follow the functions. Essentially, the research does not take into account of the actual rough surface morphology characteristics of the machined surface. In the analysis of rough surface contact deformation, most scholars simplify the contact between two rough surfaces to the rigid plane and the equivalent rough plane. However, when the two rough surfaces come into contact, the contact between the asperities is much more complicated. Therefore, under the existing assumption, the calculated value of the mechanical interface contact characteristic parameter model has a certain degree of deviation compared with the actual value.

\section{Acknowledgments}

This work was financially supported by Natural Science Foundation of Hubei Province (2017CFB759), Fundamental Research Funds for the Central Universities Project (2662017JC024), National university student innovation project (20170504094, 2017BC027, 2015310200731).

\section{References}

1. A.Greenwood, B.Williamson. Contact of nominally $f$ lat surfaces. Proc Roy Soc Lond (1966) 300-319

2. A.Majumdar, B.Bhushan. Role of Fractal Geometry i n Roughness Characterization and Contact Mechanic s of Surfaces. Journal of Tribology 112 (1990) 205-2 16

3. A.Majumdar, B.Bhushan. Fractal Model of Elastic-P1 astic Contact Between Rough Surfaces. Journal of Tr ibology 113 (1991) 1-11

4. H.L. Tian. Dynamic modeling of virtual materials in fixed joint part of mechanical structure. Huazhong $u$ niversity of science and technology (2011)
5. S.R. Ge, H. Zhu. Fractal of tribology. Mechanical in dustry press (2005)

6. H. Zhu, S.R. Ge. Study on the characterization of the surface topography of friction pairs during wear pro cess with fractal theory. Science In China 44 (2001) 259-262

7. L. He, J. Zhu. Development and development of roug h surface contact fractal model. Journal of tribology (1996) 88-97

8. W.W. Liu. Study on the contact characteristics of me chanical interface based on fractal theory. Huazhong university of science and technology (2016)

9. H.J. Chen. Digital signal processing (version 2). Hig her education publishing house (2008)

10. H. Zhu, S.R. Ge. Experimental study on the character ization of worn surface topography with characteristi c roughness parameter. Wear 255 (2003) 309-314

11. X.L. Zhang, Y.M. Huang, Y. Han. Contact stiffness model based on contact fractal theory. China mechan ical engineering (2000)

12. H. Zhu, S.R. Ge. Comparison of structure function a nd the effect of fractal characterization of RMS. Jour nal of China mining university (2004)

13. H. Zhu, S.R. Ge, Chen Guoan. Fractal characterizatio $\mathrm{n}$ of surface morphology changes. Journal of mechan ical engineering (2001)

14. X.L. Zhang, S.H. Wen. Fractal model of contact stiff ness based on contact fractal theory. Journal of agric ultural machinery (2002)

15. S.H. Wen, X.L. Zhang, M.X. Wu, X.G. Wen, P.Y. W ang. Establishment and simulation of contact stiffnes $\mathrm{s}$ fractal model by combining surface method. Journ al of agricultural machinery 40 (2009) 197-202

16. S.H. Wen, X.L. Zhang, X.G. Wen, P.Y. Wang, M.X. $\mathrm{Wu}$. Establishment and simulation of the fractal mod el of contact stiffness in combination with surface ta ngential stiffness. Journal of agricultural machinery 40 (2009) 23-227

17. W.J. Pan, X.P. Li. A normal contact stiffness fractal prediction model of dry-friction rough surface and ex perimental verification. European Journal of Mecha nics A/Solids 66 (2017) 94-102

18. S.Y. Jiang, Y.J. Zheng. A Contact Stiffness Model of Machined Plane Joint Based on Fractal Theory. Jour nal of Tribology 132 (2010) 1-7

19. X.L. Zhang, H.Q. Ding, G.S. Lan, S.H. Wen, Z.Y. Z hang, N.S. Wang. Model of contact damping and los s factor based on the combination of fractal theory. $J$ ournal of agricultural machinery 44 (2013) 287-294

20. X.L. Zhang, N.S. Wang. Tangential Damping and its Dissipation Factor Models of Joint Interfaces Based on Fractal Theory With Simulations. Journal of Trib ology 136 (2014) 1-10

21. S.R. Ge. Research on fractal features and fractal expr essions of rough surfaces. Journal of tribology (1997) 74-81 